Brazilian Journal

of Chemical

\title{
ENHANCED PRODUCTION OF PULLULAN BY TWO STRAINS OF $A$. pullulans WITH DIFFERENT CONCENTRATIONS OF SOYBEAN OIL IN SUCROSE SOLUTION IN BATCH FERMENTATIONS
}

\author{
R. F. Sena ${ }^{1 *}$, M. C. Costelli ${ }^{1}$, L. H. Gibson ${ }^{2}$ and R. W. Coughlin ${ }^{2}$ \\ ${ }^{1}$ Department of Chemical Engineering and Food Engineering, EQA, Federal University of Santa Catarina UFSC, \\ Phone: + (55) (48) 3334-9448 ext. 240, Fax: $+(55)$ (48) 3331-9687, Center of Technology, CTC, \\ PO. Box 476, Zip Code 88040-900, Florianópolis - SC, Brazil. \\ E-mail rennio@enq.ufsc.br \\ ${ }^{2}$ Department of Chemical Engineering, University of Connecticut (Uconn), \\ Storrs, CT, 06269, USA
}

(Received: July 27, 2005 ; Accepted: June 28, 2006)

\begin{abstract}
Aureobasidium pullulans is a microorganism that produces pullulan (homopolysaccharide) extracellularly through a fermentation process with sugars (maltose, d-xylose, sucrose and starch) as its carbon source. Pullulan is a linear polysaccharide of D-glycopyranose containing $(1 \rightarrow 4)-\alpha$ and $(1 \rightarrow 6)-\alpha$ linkages at a 2:1 ratio, is highly soluble in water and has various applications in the food, packaging, film and pharmaceutical industries. Lipids, primarily oils, having antifoaming properties as well as nutritional particularities, are considered an essential additional carbon source for the growth of microorganisms, especially fungi. These nutrient sources are very important for the maintenance of microorganism cells. In fact, these positive effects are only achieved when the right source is added at both the right time and the right dosage into the broth of the fermentation process.

In this research on pullulan production with the strains NRRL Y-6220 and NRRL Y-2311-1, it was found that the latter strain achieved better results for undesirable pigment formation, pullulan titer, time of maximum production (96 hours) and biomass yields than strain NRRL Y-6220, which also showed suitable results for biomass yields and cell morphology. However, the dark pigmentation of the strain NRRL Y-6220, formed through the process, makes its application unacceptable for foods and pharmaceuticals. Strain NRRL Y-23111 was shown to be a promising potential industrial microorganism, whose applications should be studied more in depth.

Keywords: Aureobasidium pullulans; Soybean oil; Sucrose; Pullulan.
\end{abstract}

\section{INTRODUCTION}

For the development of an effective and efficient fermentation process, the design of the fermentation medium is critical, due to the fact it affects fermentation yield and volumetric productivity as well as process costs. Microorganisms require carbon, nitrogen and minerals for growth and metabolism.

Lipids and oils are considered essential components of many fermentation media since they have defoaming properties and serve as a supplemental nutrient source for growth and also maintenance of the microbial cells (Ohta et al.,

*To whom correspondence should be addressed 
1995). Some authors have reported optimum results when oil was added frequently during fermentation as a sole carbon source (Gibson and Coughlin, 2002; Jones and Porter, 1998; Ohta et al., 1995).

Pullulan is a linear, water-soluble homopolysaccharide composed of maltotriose units connected by $(1 \rightarrow 6)-\alpha$ linkages. The chemical and physical properties of pullulan make it suitable for a wide range of potential applications in the food, cosmetic and pharmaceutical industries (Catley, 1973). This polysaccharide is synthesized under aerobic conditions by various species of the dimorphic yeast-like fungus Aureobasidium pullulans. The microorganism uses sugar substrates as the main carbon source for cell growth and biosynthesis of pullulan, the latter being produced mainly when growth slows down and after cessation of cell growth, i.e., when nitrogen availability is the limiting factor (Audet et al., 1996).

Fermentation can be affected by different types of carbon and nitrogen sources and results in varying pullulan yields during the process, since the phylamentous forms or chlamydospores are less productive than the yeast or pigment-free blastospores (Audet et al., 1996). The pigmentation that accompanies the morphogenetic changes to mycelia also impedes the downstream recovery steps to obtain pure polysaccharide (Pollock et al., 1992). According to Shabtai and Mukmenev (1995), the yeast-like cells did not produce much pullulan in the presence of pigment, and the nonpigmented swollen blastospores or germinating blastospores help to trigger the elaboration of pullulan or to increase its rate of elaboration, as previously suggested by Catley (1973).

In this article we describe the Aureobasidium pullulans fermentation process using in addition to sucrose as a carbon source, refined soybean oil (SBO) at different concentrations both to increase and to improve pullulan production. To avoid the black pigment formed by some strains, the strains NRRL Y2311-1 and NRRL Y-6220 were used; according to Gibson and Coughlin (2002) and Badr-Eldin et al. (1994), both produce the pigment-free pullulan and the highest yields. The process was also studied by the timing yield in hours to quantify the overall sucrose consumption for evaluation of the highest production, due to the self-consumption of the cells during the process in the absence of substrate.

Pullulan can be used as a noncaloric food ingredient and its films are water soluble and impervious to oxygen, so it can be used as a suitable packaging and coating material for foods and pharmaceuticals (Robyt, 1998; Young and Lovell, 1991). Its addition to foods prevents drying and the retrogradation of starch, and the incorporation of this biopolymer into food is reported to improve taste, flavor and texture, as well as to protect against oxidation (Shabtai and Mukmenev, 1995).

\section{MATERIALS AND METHODS}

\section{Microorganisms and Media}

Two strains of A. pullulans (NRRL Y-2311-1 and NRRL Y-6220) were kindly provided by Dr. C. P. Kurtzmann of the National Center for Agricultural Utilization Research, U.S. Department of Agriculture.

The primary medium used in this work was a complex nitrogen medium containing the following ingredients in grams/liter of water: $5 \mathrm{~g} \mathrm{KH}_{2} \mathrm{PO}_{4}, 1 \mathrm{~g}$ $\mathrm{NaCl}, 0.6 \mathrm{~g}\left(\mathrm{NH}_{4}\right)_{2} \mathrm{SO}_{4}, 0.2 \mathrm{~g} \mathrm{MgSO}_{4} \cdot 7 \mathrm{H}_{2} \mathrm{O}, 1 \mathrm{~g}$ yeast extract, $0.01 \mathrm{~g} \mathrm{FeSO}_{4}, 0.01 \mathrm{~g} \mathrm{MnSO}_{4}$ and 0.01 g $\mathrm{ZnSO}_{4}$, which was autoclaved at $121^{\circ} \mathrm{C}$ for 20 minutes (Pollock et al., 1992).

The microorganisms were stored on complex nitrogen agar plates with $50 \mathrm{~g} / \mathrm{L}$ sucrose at $8^{\circ} \mathrm{C}$. Precultures were prepared on complex nitrogen agar plates and grown at $26^{\circ} \mathrm{C}$ in $5 \mathrm{~mL}$ of malt extract broth consisting of $20 \mathrm{~g}$ malt extract, $20 \mathrm{~g}$ glucose and $1 \mathrm{~g}$ peptone per liter of water (ATTC Medium 325). These precultures were used to propagate inoculum cultures in complex nitrogen broth containing $50 \mathrm{~g} / \mathrm{L}$ sucrose. A $5 \%(\mathrm{v} / \mathrm{v})$ inoculum was used for each fermentation.

\section{Addition of Oil and Dosage Level}

The soybean oil (SBO) was added were made prior to inoculation, i.e., after the transfer of the complex nitrogen medium and before fermentation began. The dosages of SBO for both strains were 1.0, 2.0, 3.0, 4.0 and $5.0 \%(\mathrm{v} / \mathrm{v})$, and a no-oil sample was also used to evaluate its effectiveness.

\section{Shake-Flask Experiments}

The shake-flask fermentations were carried out using sucrose at $50 \mathrm{~g} / \mathrm{L}$ as the carbon source. The $\mathrm{pH}$ of the medium was 7.0, and the effect of the soybean oil (SBO) on pullulan production was investigated by altering the concentration of determined volumes of SBO per volume of medium. Shake-flask experiments were performed in $500-\mathrm{mL}(100 \mathrm{~mL}$ medium) Erlenmeyer flasks placed in a rotary shaker at $26^{\circ} \mathrm{C}$ and $250 \mathrm{rpm}$ for 120 hours. Samples of 10 $\mathrm{mL}$ were withdrawn aseptically for the analyses. 


\section{Sample Processing Procedures and pullulan Production}

Ten-ml samples of culture broth were taken at 24hour intervals. The samples were centrifuged at $10,000 \mathrm{rpm}$ for 15 minutes at room temperature to separate biomass and the decanted supernatant. Pullulan was precipitated from the supernatant using one volume of neat 2-propanol per volume of supernatant. The liquid was decanted and the precipitated pullulan was redissolved in deionized water. The resulting solution was sparged with nitrogen for approximately one hour to remove residual alcohol. The samples were then frozen at $10^{\circ} \mathrm{C}$. Once frozen solid, the pullulan solution was lyophilized to constant weight.

The biomass pellet from the initial centrifugation was resuspended in deionized water and centrifuged at $10,000 \mathrm{rpm}$ for 5 minutes. The cells were transferred to an aluminum weighting pan and dried to constant weight (approximately two hours) in a vacuum oven at $80^{\circ} \mathrm{C}$. The results are shown as cell dry weight (in g/L) (Shabtai and Mukmenev, 1995).

\section{Assays}

Sugar concentration in the culture broth samples was measured after centrifugation, but before precipitation of the pullulan with YSI Model 27 glucose analyzer using a sucrose membrane as appropriate.

Pullulan samples were assayed for protein contamination with a bicinchoninic acid (BCA) protein assay (Pierce Chemical Company, Rockford, IL, USA) and for total carbohydrate concentration using phenolsulfuric acid assay (DuBois et al., 1956).

Purity of produced pullulan was estimated by enzymatic hydrolysis to maltotriose with pullulanase (Sigma E.C.3.2.1.41) from Klebsiella pneumoniae (Leathers et al., 1998) followed by determination of maltotriose reducing sugar equivalents using the dinitrosalisylic acid (DNS) method (Miller, 1959).

\section{RESULTS}

\section{Comparison of Strains}

Figures 1 and 2 are plots of the time course of a typical fermentation showing the relationships among substrate concentration, biomass production and pullulan production. Total depletion of the sugar substrate occurs after about three days and a maximum pullulan concentration, at about three to five days. The decreasing pullulan concentration appears to be associated with increasing biomass concentration. This suggests the pullulan is being hydrolyzed and utilized by the microorganism after the substrate is consumed.

An initial medium concentration of $50 \mathrm{~g} / \mathrm{L}$ sucrose was depleted in three days and the maximum pullulan titer occurred at the fourth day of fermentation. The figures show that sucrose is consumed by the third day of fermentation, when the self-consumption of the pullulan cells probably starts. When the source for the cell maintenance decreases at the fifth day of the process, the higher yield was attained.

The two different strains of the fungus were compared for the amount of pullulan produced, the molecular weight distribution and the extent of production of the undesirable pigment. Table 1 summarizes the results on production of pullulan, biomass, pigment formation and pullulan yield.

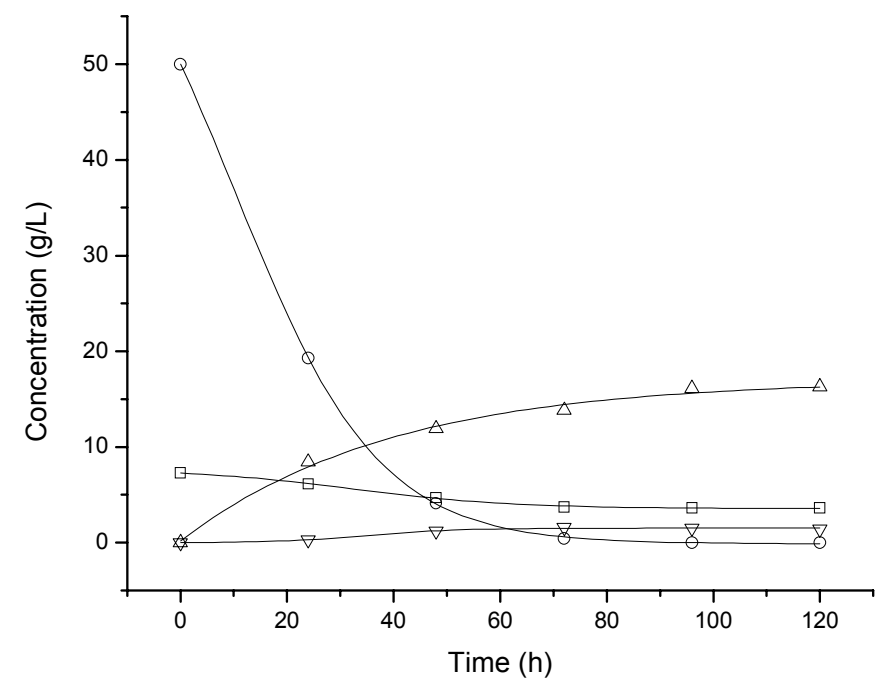

Figure 1: Time course for batch fermentation of $A$. pullulans NRRL Y-2311-1: pH ( $\square$ ), Cell Dry Weight $(\nabla)$, Pullulan Dry Weight $(\Delta)$ and Sucrose Concentration $(\circ)$. 


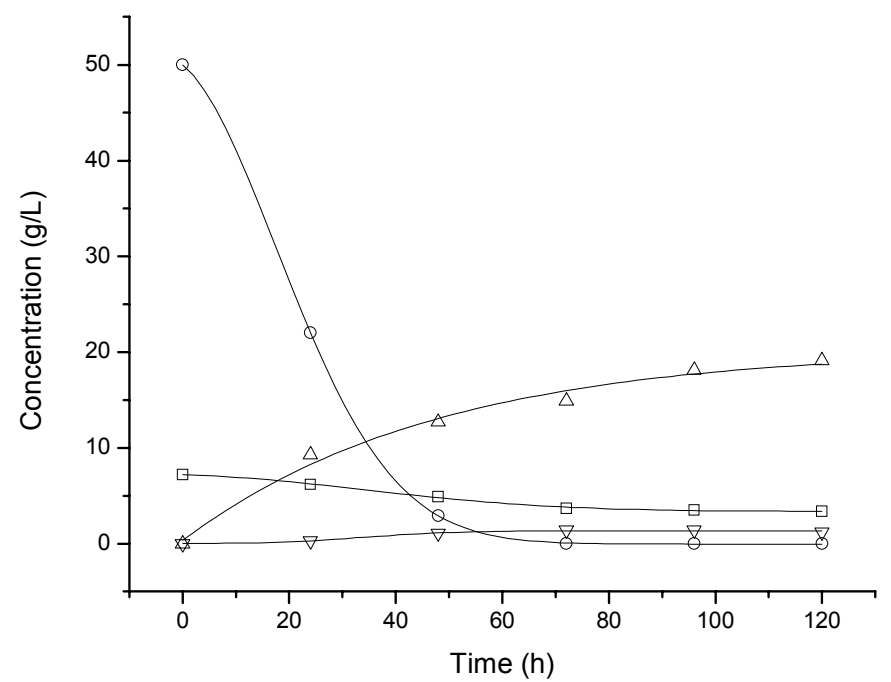

Figure 2: Time course for batch fermentation of A. pullulans NRRL Y-6220: $\mathrm{pH}(\square)$, Cell Dry Weight $(\nabla)$, Pullulan Dry Weight $(\Delta)$ and Sucrose Concentration $(\circ)$.

Table 1: Maximum pullulan production, biomass production, pigment formation and pullulan yield calculations for two strains of $\boldsymbol{A}$. pullulans studied using an initial sucrose concentration of $50 \mathrm{~g} / \mathrm{L}$ during the preliminary assays

\begin{tabular}{|l|c|c|}
\hline \multirow{2}{*}{ Parameters } & \multicolumn{2}{|c|}{ Strains of Aullulans } \\
\cline { 2 - 3 } & NRRL Y-2311-1 & NRRL Y-6220 \\
\hline Maximum Pullulan, Titer (g/L) & 26.24 & 17.40 \\
Time of Maximum, Pullulan Titer (h) & 96 & 120 \\
Biomass (g/L) & 6.91 & 11.02 \\
Formation of Pigment & None & Dark \\
Appearance of Pigment (h) & None & 72 \\
Pullulan Yield, (g product/g substrate) & 0.525 & 0.348 \\
\hline
\end{tabular}

Strain NRRL Y-2311-1 was the strain with the higher yield, as well as the one that produced the less biomass and had no pigment formation; the latter characteristic is the most important for both pharmaceutical and food applications. Maximum pullulan production occurred at 96 hours. The $\mathrm{pH}$ of the fermentation broth fell from 6.5 to 3.5 within the first 24 hours and stayed near 3.5 for the remainder of the fermentation. Yeast-like cells were the dominant morphology up to day 3 , when mycelial growth and chlamydospores began to appear.

For strain NRRL Y-6220, maximum pullulan production occurred at day 5 . The $\mathrm{pH}$ of the fermentation broth fell from 6.5 to only 5.0 within the first 24 hours and remained near 4.0 through day 5. Cell morphology was essentially mycelial from day 1 onwards. Pigment formation began on day 3 and turned the fermentation broth completely dark by day 5 .

\section{Purity of the pullulan Product}

Table 2 summarizes the results of the assays performed on the pullulan samples for total protein and carbohydrate contents. The last column of Table 2 lists the weight percentage of each sample found to be maltotriose following extensive enzymatic hydrolysis with pullulanase $(0.1 \%(\mathrm{w} / \mathrm{v})$ pullulan in sodium acetate buffer, $\mathrm{pH} 5.0,0.1 \mathrm{U} / \mathrm{ml}$ pullulanase, 48 hours, $26^{\circ} \mathrm{C}$ ). Similar data for pullulan obtained from Sigma Chemical Co. (Catalog \#p-4516) is also included for comparison.

As can be observed in Table 2, the pullulan produced by the more productive strain (A. pullulans NRRL Y-2311-1) was recovered in reasonably pure form. The amount of protein in the samples was too small to explain deviation of carbohydrate concentrations from $100 \%$. Some of this deviation may be caused by pigment in some of the samples. 
Table 2: Summary of protein, carbohydrate and maltotriose content of pullulan samples

\begin{tabular}{|l|c|c|c|}
\hline \multirow{2}{*}{ Sample } & \multicolumn{3}{|c|}{ Concentrations (w/v) } \\
\cline { 2 - 4 } & Protein* & Carbohydrate* & Maltotriose* $^{*}$ \\
\hline NRRL Y-6220 & $0 \%$ & $64 \%$ & $91 \%$ \\
NRRL Y-2311-1 & $0 \%$ & $96 \%$ & $96 \%$ \\
Sigma Chemical Reference Sample & $0 \%$ & $94 \%$ & $97 \%$ \\
\hline
\end{tabular}

* Concentration measured by assay divided by concentration of pullulan sample assayed multiplied by 100 .

\section{Effect of SBO on pullulan Productivity}

For the process of fermentation, the $\mathrm{pH}$, percentage of SBO and pullulan production of the $A$. pullulans were compared. In Tables 3 and 4 the results for 72, 96 and 120 hours of fermentation are summarized.

The samples with $5.0 \%$ soybean oil obtained the best results of pullulan production for both strains, showing the effect of vegetable oils as an additional carbon source on pullulan production at an acid $\mathrm{pH}$, as also shown in Figures 3 and 4.
The data in Tables 3 and 4 also show that the use of soybean oil as a sole carbon source is not an effective substrate for the production of pullulan, or of biopolymers in general, due the unavailability of sugars for conversion by the microorganism. Thus, the yield of pullulan can be enhanced by the addition of vegetable oils to the sugar substrate, as shown in Figures 3 and 4. The best dosage requires further research, mainly for industrial processes, because of the cost of this additional dosage and to avoid an increase in fermentation viscosity, which should be studied to evaluate the procedures and how to handle it.

Table 3: Pullulan production from different percentages of soybean oil by $A$. pullulans strain NRRL Y-2311-1

\begin{tabular}{|c|c|c|c|c|}
\hline \multirow{2}{*}{ Oil (\%) } & \multirow{2}{*}{$\mathbf{p H}$} & \multicolumn{3}{|c|}{ Pullulan Production (g/L) } \\
\cline { 3 - 5 } & & $\mathbf{7 2 h}$ & $\mathbf{9 6 h}$ & $\mathbf{1 2 0 h}$ \\
\hline 0.0 & 3.7 & 15.46 & 19.04 & 18.66 \\
1.0 & 4.2 & 18.54 & 21.26 & 19.14 \\
2.0 & 4.3 & 18.99 & 22.24 & 21.50 \\
3.0 & 4.4 & 19.73 & 27.04 & 25.12 \\
4.0 & 4.5 & 20.35 & 27.02 & 25.98 \\
5.0 & 4.4 & 23.05 & 29.58 & 28.04 \\
SBO & 6.9 & $* *$ & $* *$ & $* *$ \\
\hline
\end{tabular}

* Soybean oil as a sole carbon source at $50 \mathrm{~g} / \mathrm{L}$.

** No pullulan production.

Table 4: Pullulan production from different percentages of soybean oil by $A$. pullulans strain NRRL Y-6220

\begin{tabular}{|c|c|c|c|c|}
\hline \multirow{2}{*}{ Oil (\%) } & \multirow{2}{*}{$\mathbf{p H}$} & \multicolumn{3}{|c|}{ Pullulan Production (g/L) } \\
\cline { 3 - 5 } & & $\mathbf{7 2 h}$ & $\mathbf{9 6 h}$ & $\mathbf{1 2 0 h}$ \\
\hline 0.0 & 3.5 & 16.27 & 19.40 & 19.01 \\
1.0 & 4.4 & 17.87 & 25.28 & 21.87 \\
2.0 & 4.5 & 18.81 & 25.98 & 24.67 \\
3.0 & 4.6 & 18.98 & 26.36 & 25.90 \\
4.0 & 4.6 & 19.12 & 26.88 & 25.96 \\
5.0 & 4.7 & 21.92 & 27.48 & 26.26 \\
SBO* & 6.5 & $* *$ & $* *$ & $* *$ \\
\hline
\end{tabular}

* Soybean oil as a sole carbon source at $50 \mathrm{~g} / \mathrm{L}$.

** No pullulan production. 


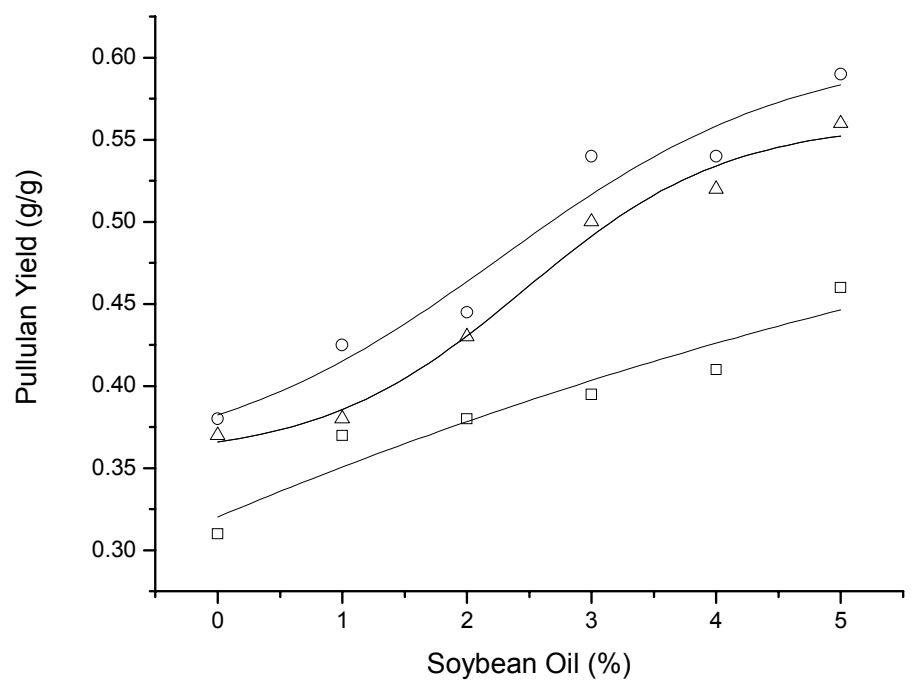

Figure 3: The effect of SBO on pullulan yield (g product/g substrate) using strain

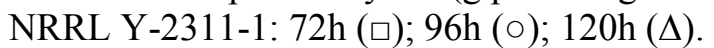

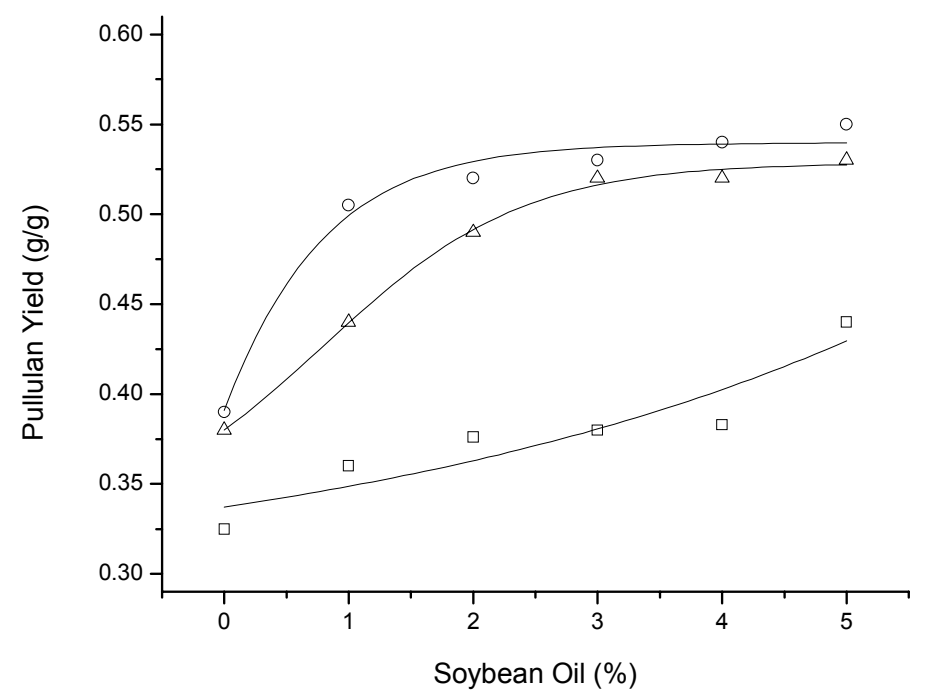

Figure 4: The effect of SBO on pullulan yield (g product/g substrate) using strain NRRL Y-6220: 72h (口); 96h (०); 120h ( $\Delta)$.

The $\mathrm{pH}$ of the samples was kept above 4.0 for the strains to which oil had been added, the ones that had the best results. When oils were used as a carbon source, there were not as many differences in cell growth; however, because of oil consumption, unsaturated fatty acids accumulated, which resulted in a decrease in the $\mathrm{pH}$ of the culture broth (Park et al., 1994). For this reason, further research should be conducted to evaluate how $\mathrm{pH}$ is related to enhancement of pullulan production.

However, as can be observed in Tables 3 and 4, the $\mathrm{pH}$ was measured throughout the whole fermentation process, but only the average $\mathrm{pH}$ results were reported. In fact, the deviation of the data was low enough to suggest constant $\mathrm{pH}$ values for each process at each initial sugar concentration and addition of oil.

According to Badr-Eldin et al. (1994), pullulan production is favored by a high $\mathrm{C} / \mathrm{N}$ ratio and an initial $\mathrm{pH}$ between 6.5 and 7.5. In fact, Schuster et al. (1993) reported lower polysaccharide productivity at a $\mathrm{pH}$ between 2.0 and 4.0, when pullulan production seemed to commence upon reaching ammoniumlimiting conditions.

On the one hand, strain NRRL Y-2311-1 with $5.0 \%$ soybean oil achieved the best results for pullulan production; on the other hand, strain NRRL Y-6220 achieved better results for pullulan production, on average. The highest pullulan production was achieved at 96 hours of fermentation, 
which is, according to Simon et al. (1995), due to cell consumption in the absence of substrate, where the sucrose was entirely consumed by the third day of the process.

Some of the SBO was simultaneously consumed with the sugar, and its concentration in the broth consequently decreased. Marked morphogenetic changes were detected in cultures with both substrates. Filamentous growth and yeast-like cells dominated these cultures, possibly because sucrose was present from the beginning of the growth phase. Previous reports (Gibbs and Serviour, 1998; Schuster et al., 1993) indicated that certain nitrogen sources, e.g. the amino acids glutamine or glutamic acid, suppress the morphogenetic shift from yeast to filamentous. Accordingly we examined the effects of several nitrogen sources on the mutagenesis of $A$. pullulans when grown on sucrose (not shown herein).

\section{DISCUSSION}

Different strains of $A$. pullulans produce substantially different titers and yields (on sugar substrates) of pullulan (Campbell et al., 2004; Gibson and Coughlin, 2002; West and Strohfus, 2001). Compared to the other strain, the NRRL Y2311-1 produced a higher yield of unpigmented and reasonably higher molecular weight pullulan when cultivated under the conditions reported herein. Those behaviors were better achieved as the percentage of refined soybean oil was increased throughout the samples; however, due to the larger amount of oil per liter in the broth, the high viscosity was an evident hindrance in pullulan precipitation due to both cell impregnation on the flask walls and the foaming property acquired by the broth.

In fact, subsequent studies, as well this one, reported that $A$. pullulans produces pullulan with better results for titer and yields for sucrose concentrations of around $50 \mathrm{~g} / \mathrm{L}$, and the sucrose was totally consumed by the end of the third day of process, on average (Gibson and Coughlin, 2002; Mohammad et al., 1995b; Leathers et al., 1988).

In strain NRRL Y-6220 an undesirable dark pigment formed during the process, which discourages its applications to foods and pharmaceuticals. Therefore, some studies are being conducted using chemical treatment (ethidium bromide) and UV light mutagenesis techniques on original strains to eliminate melanin production.

The molecular weight distribution of pullulan produced by strain NRRL Y-2311-1 shifted towards a lower molecular weight from day 1 through day 4 . The shift toward low molecular weight with time is similar to behavior reported by Madi et al. (1996).

This change in the molecular weight distribution over time may be caused by pullulanase production that is linked to the availability of the carbon and energy source. High sucrose concentration could inhibit production of pullulanase. This suggests that the lower molecular weight fractions of pullulan are disappearing, possibly by catabolism of $A$. pullulans. Pullulan harvest at the time of maximum production for each strain had either a bimodal or polymodal distribution of molecular weights. The ranges of pullulan molecular weight determined in this study are in reasonable agreement with those reported by Youssef et al. (1999), Kim et al. (1998), and Madi et al. (1996).

Utilization of refined soybean oil (SBO) by $A$. pullulans had not been reported previously. It requires a large oil droplet surface for growth, even when the oil is abundant in the culture. It requires both the induction of an active lipolytic system and the production of biosurfactant(s) in the culture. These facts suggest that in the initial stage of fermentation the increase in the $\mathrm{C} / \mathrm{N}$ ratio of the cells is low, on the one hand, and pullulan productivity is low on the other, as previously reported by Youssef et al. (1999).

The protein, carbohydrate and maltotriose contents determined in this study for strain NRRL Y2311-1 are in reasonable agreement with the reference used for comparison.

\section{ACKNOWLEDGEMENTS}

The authors are grateful to the Department of Chemical Engineering of the Uconn. Special acknowledgements go to R. Vinopal and R. Vieth for their constructive remarks that provided helpful suggestions and discussions.

\section{REFERENCES}

Audet, J., Lounes, M., and Thibault, J., Pullulan fermentation in a reciprocating plate bioreactor. Bioprocess Engineering, No. 15, 209-214 (1996).

Badr-Eldin, S.M., El-Tayeb, O.M., El-Masry, H.G., Mohammad, F.H.A. and Abd El-Rahman, O.A., Polysaccharide production by Aureobasidium pullulans: factors affecting polysaccharide formation. World Journal of Microbiology and Biotechnology, No. 10, 423-426 (1994). 
Campbell, B.S., Siddique, A.M., McDougall, B.M. and Serviour, R.J., Which morphological forms of the fungus Aureobasidium pullulans are responsible for pullulan production? FEMS Microbiology Letters, No. 232, 225-228 (2004).

Catley, B.J., The rate of elaboration of the extracellular polysaccharide pullulan during growth of Pullularia pullulans. Journal of General Microbiology, No. 78, 33-38 (1973).

Catley, B.J., Pullulan, a relationship between molecular weight and fine structure. FEBS Letters, No. 10, 190-193 (1970).

DuBois, M., Gilles, K.A., Hamilton, J.K., Rebers, P.A. and Smith, F., Colorimetric method for determination of sugars and related substances. Analytical Chemistry, No. 28, 350-356 (1956).

Gibbs, P.A. and Serviour, R.J., Does the agitation rate and/or oxygen saturation influence exopolysaccharide production by Aureobasidium pullulans in batch culture? Applied Microbiology and Biotechnology, No. 46, 503-510 (1996).

Gibbs, P.A. and Serviour, R.J., The production of exopolysaccharides by Aureobasidium pullulans in fermenter with low-shear configurations. Applied Microbiology and Biotechnology, No. 49, 168-174 (1998).

Gibson, L.H. and Coughlin, R.W., Optimization of high molecular weight pullulan production by Aureobasidium pullulans in batch fermentations. Biotechnology Progress, No. 18, 675-678 (2002).

Israilides, C.J., Smith, A., Harthill, J.E., Barnett, C., Bambolow, G. and Scanlon, B., Pullulan content of the ethanol precipitate from fermented agroindustrial wastes. Applied Microbiology and Biotechnology, No. 49, 613-617 (1998).

Jones, A.M. and Porter, M.A., Vegetable oils in fermentation: beneficials effects of low-levels supplementation. Journal of Industrial Microbiology and Biotechnology, No. 21, 203-207 (1998).

Kim, J.H., Kim, M.R., Lee, J.H. and Lee, J.W., Production of high molecular weight pullulan by Aureobasidium pullulans using glucosamine. Biotechnology Letters, No. 49, 613-617 (1998).

Leathers, T.D., Nofsinger, G.W., Kurtzman, C.P. and Bothast, R.J., Pullulan production by color variant strains of Aureobasidium pullulans. Journal of Industrial Microbiology, No. 3, 231239 (1998).

Madi, N.S., McNell, B. and Harvey, L.M., Influence of culture $\mathrm{pH}$ and aeration on ethanol production and pullulan molecular weight by Aureobasidium pullulans. Journal of Chemical Technology and Biotechnology, No. 66, 343-350 (1996).
Miller, J.L., Use of dinitrosalicylic acid reagent for determination of reducing sugar. Analytical Chemistry, No. 31(3), 426-428 (1959).

Mohammad, F.H.A., Badr-Eldin, S.M., El-Tayeb, O.M. and Abd El-Rahman, O.A., Polysaccharide production by $A$. pullulans III: The influence of initial sucrose concentration on batch kinetics. Biomass and Bioenergy, No. 8, 121-129 (1995a).

Mohammad, F.H.A., Badr-Eldin, S.M., El-Tayeb, O.M., and Abd El-Rahman, O.A., Polysaccharide production by $A$. pullulans III: The influence of initial sucrose concentration on the dynamics of the fermentation. African Journal of Mycology and Biotechnology, No. 2, 61-75 (1995b).

Ohta, N., Park, Y.S., Yabiro, K. and Okabe, M., Comparison of neomycin production from Streptomyces fradiae cultivation using soybean oil as sole carbon source in an air-lift bioreactor and a stirred-tank reactor. Journal of Fermentation Bioengineering, No. 79, 443-448 (1995).

Ono, K., Yasuda, N. and Ueda, S., Effect of pH on pullulan elaboration by $A$. pullulans $\mathrm{S}-1$. Agricultural Biology and Chemistry, No. 41, 2113-2118 (1977).

Park, Y.S., Momose, Y., Tsunoda, K. and Okabe, M., Enhancement of cephamycin $\mathrm{C}$ production using soybean oil as the sole carbon source. Applied Microbiology and Biotechnology, No. 40, 773-779 (1994).

Pollock, T.J., Thorne, L. and Armentrout, R.W., Isolation of new Aureobasidium pullulans strains that produce high-molecular-weight pullulan with reduced pigmentation. Applied and Environmental Microbiology, No. 58, 877-883 (1992).

Reeslev, M. and Jensen, B., Influence of $\mathrm{Zn}^{2+}$ and $\mathrm{Fe}^{3+}$ on polysaccharide production and mycelial/yeast dimorphism of Aureobasidium pullulans in batch cultivations. Applied Microbiology and Biotechnology, No. 42, 910915 (1995).

Robyt, J.F., Essentials of Carbohydrate Chemistry. Springer-Verlag, New York (1998).

Shabtai, Y. and Mukmenev, I., Enhanced production of pigment-free pullulan by a morphogenetically arrested Aureobasidium pullulans (ATCC 42023) in a two-stage fermentation with shift from soybean oil to sucrose. Applied Microbiology and Biotechnology, No. 43, 595-603 (1995).

Schuster, R., Wenzig, E. and Mersmann, A., Production of the fungal exopolysaccharide pullulan by batch-wise and continuous fermentation. Applied 
Microbiology and Biotechnology, No. 39, 155-158 (1993).

Simon, L., Bouchet, B., Bremond, K., Gallant, D.J. and Bouchonneau, M., Studies on pullulan extracellular production and glycogen intracellular content in Aureobasidium pullulan. Canadian Journal of Microbiology, No. 44, 1193-1199 (1998).

Simon, L., Bouchet, B., Caye-Vaugien, C., Gallant, D.J. and Bouchonneau, M., Pullulan elaboration and differentiation of the resting forms in Aureobasidium pullulan. Canadian Journal of Microbiology, No. 40, 35-45 (1995).

Simon, L., Bouchet, B., Caye-Vaugien, C. and Bouchonneau, M., Relation between pullulan production, morphological state and growth conditions in Aureobasidium pullulans: new observations. Journal of General Microbiology, No. 139, 979-985 (1993).

West, T.P. and Strohfus, B., Polysaccharide production by immobilized Aureobasidium pullulans cells in batch bioreactors. Microbiology Research, No. 156, 285-288 (2001).

Young, R.J. and Lovell, P.A., Introduction to Polymers. Chapman \& Hall, New York (1991).

Youssef, F., Roukas, T. and Biliaderis, C.G., Pullulan production by a non-pigmented strain of Aerobasidium pullulans using batch and fedbatch culture. Process Biochemistry, No. 34, 355366 (1999). 